\title{
Nuances of the Composite Face-lift Technique
}

\author{
Ramsey J. Choucair, M.D., ${ }^{1}$ and Sam T. Hamra, M.D. ${ }^{1}$
}

The composite face-lift is a well-described, comprehensive facial rejuvenation technique that results in harmonious repositioning of all components of the aging face. It is distinguished by the superior-medial movement of the cheek and the septal reset maneuver. The composite rhytidectomy reverses the unbalanced vectors of the traditional face-lift in patients with the lateral sweep and may reestablish the youthful convexity in patients with hollow eyes.

KEYWORDS: Composite rhytidectomy, balanced vector, superior-medial, hollow eyes, lateral sweep, septal reset

The composite face-lift is a well-described technique for comprehensive facial rejuvenation that includes tissue repositioning of every part of the aging face. ${ }^{1}$ The most distinct characteristic separating this from all other face-lifts is the movement of the cheek and malar tissues in a superior-medial vector instead of a superior-lateral vector and arcus marginalis release and septal reset in which the septum orbitale is sutured over the orbital rim (Figs. 1-2). These two distinguishing factors (superior-medial vector of the cheek and arcus marginalis release with septal reset) are the critical maneuvers that distinguish a composite face-lift from a conventional procedure.

Conventional procedures are defined as subcutaneous or skin lifts, SMAS and deep plane or malar fat procedures. All of these face-lift techniques share similar superior-lateral tissue repositioning (Fig. 3). The superior-medial vector of the composite face-lift "balances" the superior-lateral vector of the lower face (an unopposed vector in conventional face-lifts) yielding a true, "balanced vector face-lift." This represents a logical anatomically sound and well-established technique for harmonious facial rejuvenation of the aging face.

The advantage of the composite face-lift extends to rejuvenation of the previously face-lifted patient. The "hollow eye" and "lateral sweep" are common results that appear after conventional, superior-lateral vector facelifts $^{2,3}$ (Fig. 4). An unopposed lateral vector or SMAS lift can create a strong pull on the lower face without the balancing medial vector movement of the cheek. The composite face-lift can effectively reverse these undesirable effects after a previous face-lift.

The aim of the composite face-lift is to reverse the normal course of aging, which includes the skeletonization of the periorbital area as the soft tissue changes lead to the appearance of the underlying bony anatomy (Fig. 5). The soft tissue contour of the lower eyelid becomes concave, and an "eye socket" slowly develops. The vertical height of the lower eyelid elongates, and the eyelid cheek junction becomes clearly defined. ${ }^{4}$ The composite face-lift with its arcus marginalis release and septal reset attempts to reestablish the youthful appearance of the lower eyelid cheek junction, creating a convex lower eyelid contour.

The composite face-lift technique has evolved in a well-documented fashion over 25 years when the senior author began performing the Skoog face-lift. This was followed by a modification in 1978, "The Tri-Plane Facelift," which added a preplatysmal cervical dissection separating the preplatysmal fat from the lower face. ${ }^{5}$ In 1985, the cheek fat or malar fat was added to the face-lift

\footnotetext{
${ }^{1}$ Dallas, Texas.

Address for correspondence and reprint requests: Ramsey J. Choucair, M.D., 9301 N. Central Expressway, Suite \#551, Dallas, TX 75231 (e-mail: rjc@drchoucair.com).

Contemporary Face-lift Techniques; Guest Editor, Larry H.
}

Hollier, Jr., M.D., F.A.C.S.

Semin Plast Surg 2009;23:247-256. Copyright (C) 2009 by Thieme Medical Publishers, Inc., 333 Seventh Avenue, New York, NY 10001, USA. Tel: +1(212) 584-4662.

DOI 10.1055/s-0029-1242183. ISSN 1535-2188. 


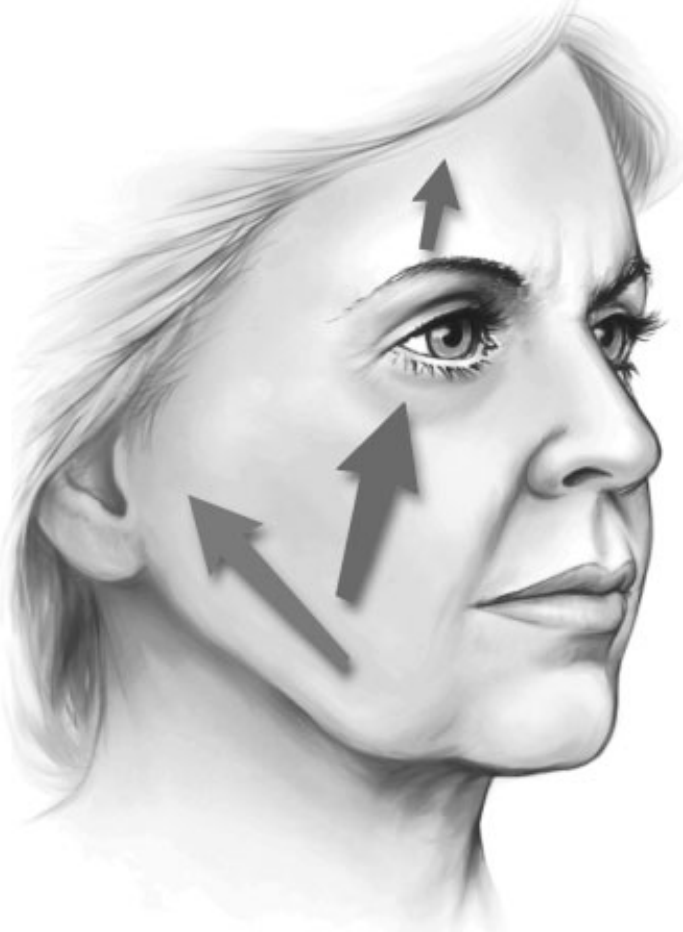

Figure 1 The composite face-lift vector is superior-medial.

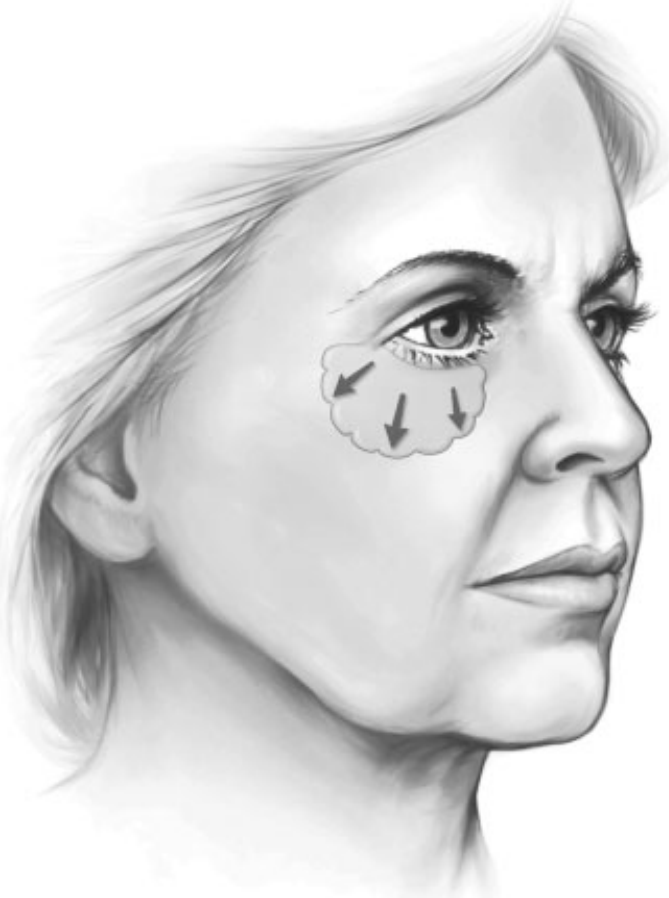

Figure 2 The composite face-lift includes a septal reset that prevents a hollow lower eyelid.

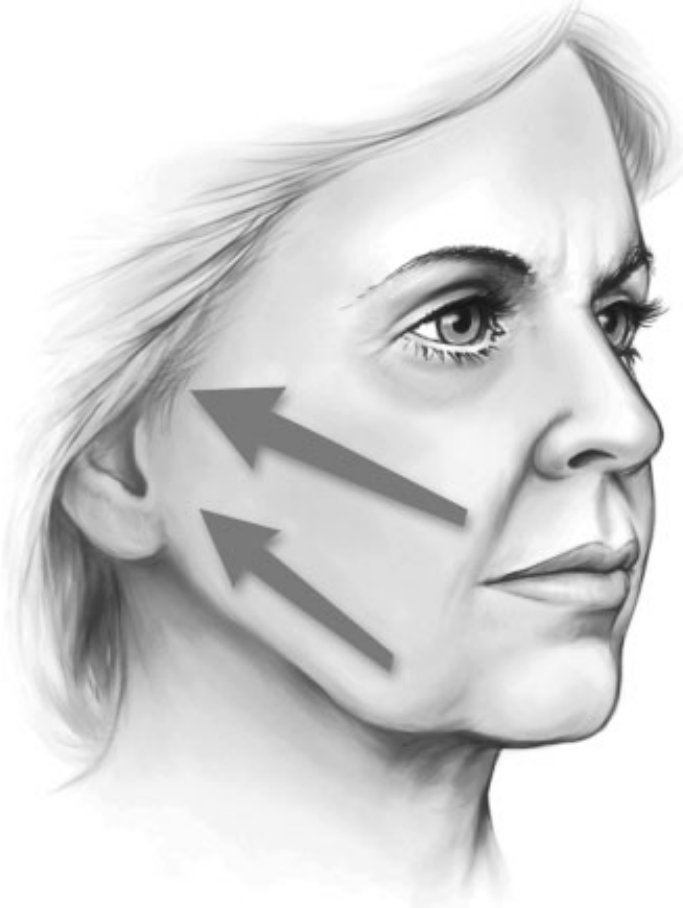

Figure 3 All conventional face-lifts are lateral vector face-lifts.

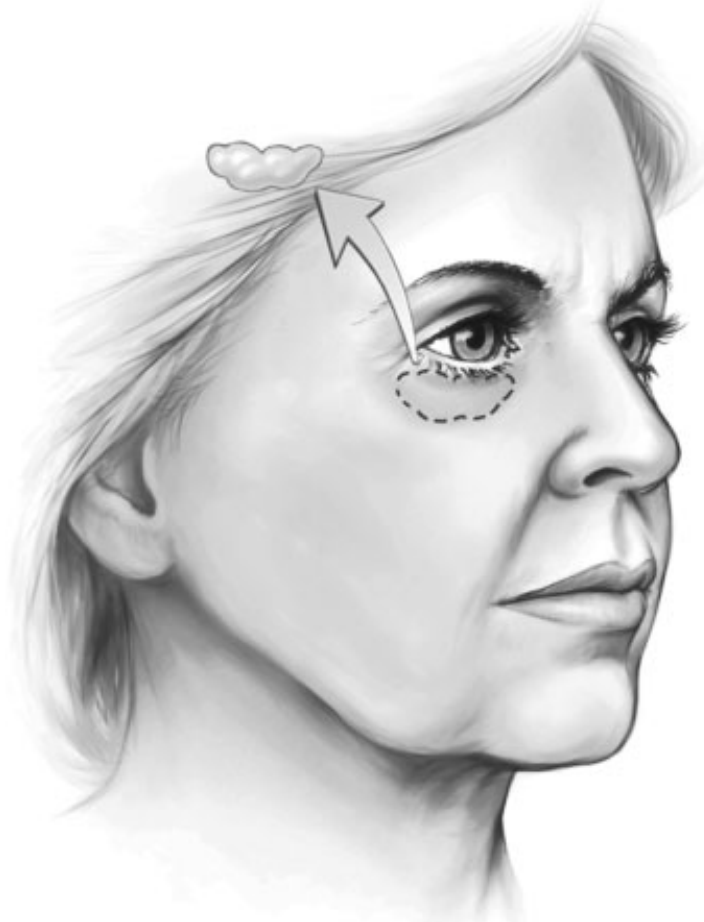

Figure 4 The traditional lower blepharoplasty usually includes orbital fat removal. 

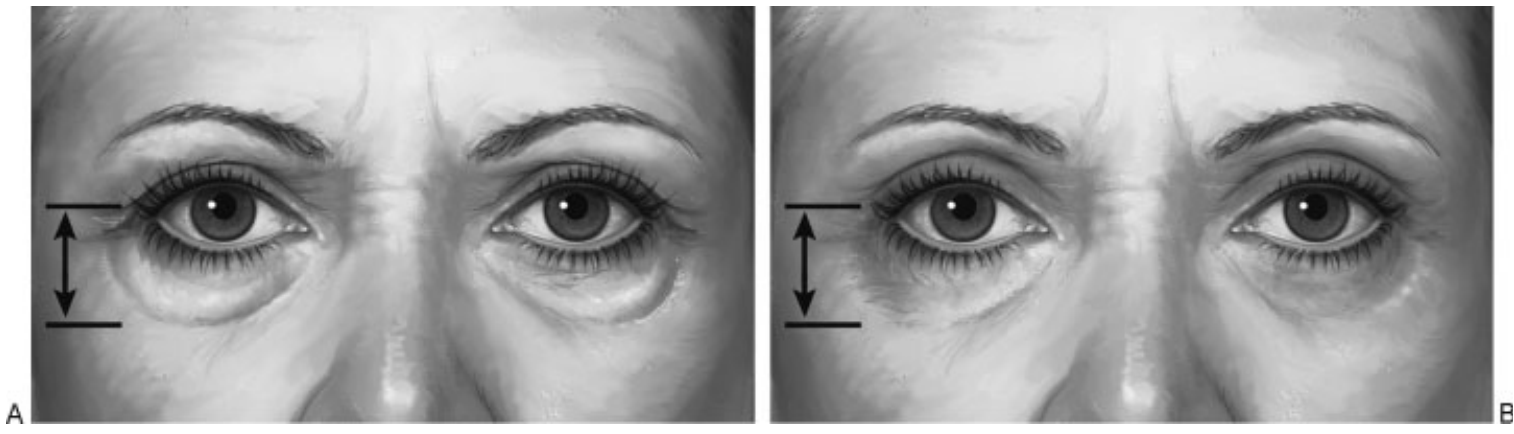

Figure 5 (A) Removal of orbital fat can (B) create a hollow lower eyelid.

flap and was published as the "Deep Plane" face-lift. ${ }^{6}$ It was not until 1990 that the orbicularis oculi was added to the face-lift flap in the first attempt to create a more youthful eyelid junction. This was the first published use of the term "composite face-lift," as the flap contained skin, muscle, and fat.

The lower eyelid fat was first manipulated by the senior author in 1991 by releasing the fat and suturing it across the orbital rim. The arcus marginalis was released with this additional maneuver although the septum orbitale was excised. ${ }^{7}$ In 1996, the technique was further modified to include the zygomaticus major muscle in the flap as well with the orbicularis oculi muscle. This was

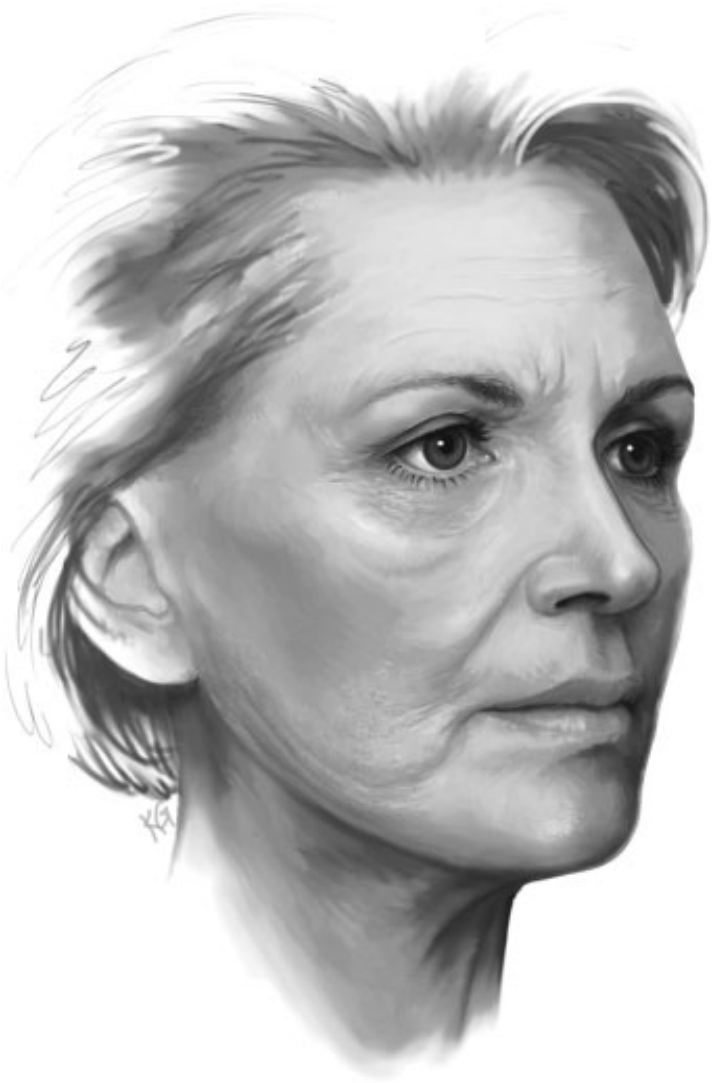

Figure 6 Lateral vector face-lift can in time produce a "lateral sweep," or pull of the lower face. published as the "Zygorbicular Dissection" for midface elevation. ${ }^{8}$ At the same time, this technique included a modification to the orbital fat transfer with the septum orbitale included with the fat transfer below the orbital rim. This was then more clearly described as the septal reset technique.

Recent additions to the technique include a more forceful elevation of the cheek mass following the septal reset with fixation of the cheek mass to the inferior orbital rim periosteum. This has created an even more youthful malar cheek complex with soft tissue auto-augmentation of the cheek. Another noteworthy change is that the original composite face-lift included a Skoog dissection ${ }^{9}$ (i.e., the skin and SMAS were moved together without separation): Because the key to harmonious facial rejuvenation is the balancing of the lateral vector of the cheek movement with a superior-medial vector of the cheek, the SMAS dissection is often irrelevant in contributing to this harmonious appearance. Therefore, a SMAS dissection is not always performed with the composite face-lift.

The advantage of composite face-lift is a more harmonious and youthful appearance without the stigma of a face-lifted or surgical appearance (Fig. 6). The disadvantage, as in all cases of advanced surgery, is the associated learning curve. A more complete rejuvenation also requires more time and surgical effort associated with a slightly longer recovery. Patients who understand the advantage of the composite lift will accept the slightly longer recovery time knowing that the results will be worthwhile, long lasting, and will avoid the "facelifted" look.

\section{INDICATIONS}

The composite rhytidectomy is ideal as a primary procedure. Lower eyelid and cheek tissue repositioning reverses the aging and inevitable skeletonization of the periorbital region (Fig. 7). The superior-medial vector of the midface movement may cause redundancy in the midface region, and therefore repositioning the temple/ forehead region may be an obligatory portion of the procedure. ${ }^{10,11}$ As a procedure for secondary face-lift, 


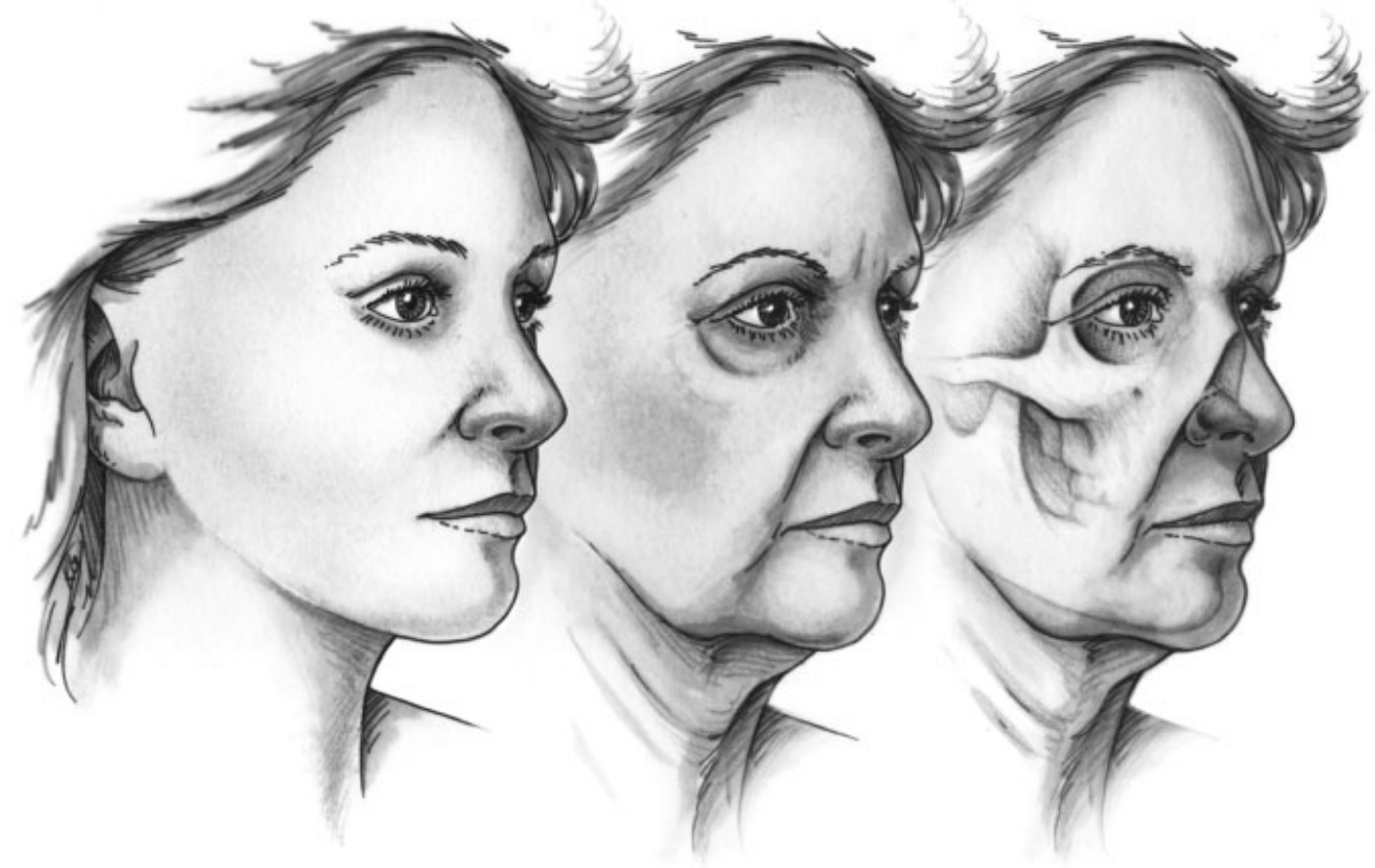

Figure 7 There is a progressive skeletonization of the periorbit, which became apparent about the end of the third decade.

the composite rhytidectomy has been shown to correct the unattractive signs after conventional face-lifts. This includes the improvement of the hollow eye after lower eyelid fat removal and the lateral sweep/pulled face often seen after conventional face-lifts that utilize only the lateral vector approach. The arcus marginalis release and septal reset combined with the superior-medial vector movement of the cheek lift may restore the natural contours of the face after the "unbalanced" pull of conventional operative techniques. ${ }^{12}$

Patients undergoing facial rejuvenation have a preoperative medical evaluation appropriate for their age and medical conditions to clear them for elective

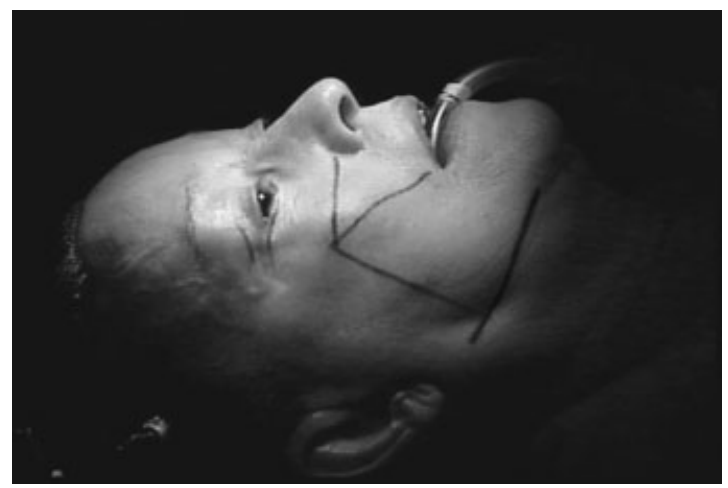

Figure 8 The markings on the face include the jawline and the zygomaticus muscles. surgery. Most procedures are done under a light general anesthetic. Intravenous sedation is rarely used.

Specific attention is given to preexisting ophthalmologic problems such as a history of dry eyes or previous eyelid surgery. Postoperative expectations for eye care including an extended period of using eye lubricant drops and protection from conditions that will dry out the eye are emphasized.

\section{PREOPERATIVE MARKINGS}

The facial topography is marked preoperatively in a sitting position (Fig. 8). This includes the mandibular border, and course the zygomaticus major/minor muscle from the malar eminence to the corner of the mouth and the alar base. These landmarks will shift in a supine operative position. The extent of the neck dissection is delineated by the anterior margin of the sternocleidomastoid muscle laterally, the sternal notch inferiorly, and the mandibular margin superiorly. The preauricular incisions for the composite rhytidectomy are essentially the same as for conventional procedures including a retrotragal incision and a postauricular incision extending along or into the postauricular hairline for exposure. A hairline forehead incision is made in the appropriate cases where the forehead is high or a coronal incision made in patients with normal forehead height. 


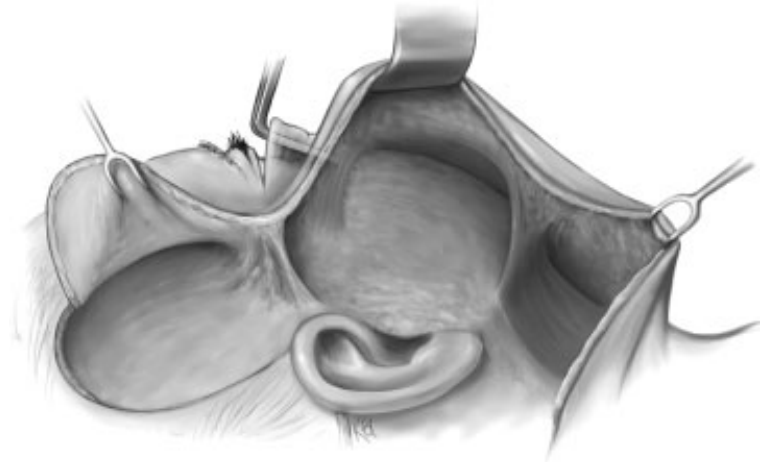

Figure 9 There are three mesenteries created on the face that contain the motor nerves.

\section{TECHNIQUE}

Composite rhytidectomy includes dissections in the following planes (Fig. 9):

1. Subcutaneous/preplatysmal neck dissection

2. Subcutaneous or sub-SMAS dissection in the lower face

3. Zygo-orbicular dissection through a subciliary/lower eyelid incision

4. Subgaleal forehead/temporal dissection

The knowledge of facial anatomy is obviously essential to any face-lift technique, and the composite face-lift is no different. The facial dissection superficial to the zygomaticus major or the deep plane is safe as the nerves in the mimic muscle into the muscle from the underside. The zygo-orbicular dissection is perhaps the most difficult to describe. One must retain a mesentery between the zygoorbicular dissection (subciliary approach) and the face-lift dissection. This mesentery contains the branches to the nerves and orbicularis muscle and is called the meso-orbicularis. Finally, the frontal branch of the facial nerve is found on the undersurface of the forehead flap and is protected by meso-temporalis the mesentery that separates the face and forehead dissection. The subgaleal dissection is obviously deep to this branch.

The surgical sequence is described as follows:

\section{Upper eyelid blepharoplasty}

- Premeasured skin and a modest strip of orbicularis muscle are removed, and medial/middle fat compartments are conservatively trimmed as indicated. Closure of upper eyelid incisions leaving the lateral one-quarter of the incision open for subsequent fixation of the lower eyelid orbicularis flap to the lateral orbital rim.
2. Neck dissection

- Subcutaneous dissection as previously mentioned, with platysma muscle identification.

3. Right cheek dissection and postauricular neck dissection

- Deep plane dissection just superficial to the zygomaticus muscle, leaving the cheek fat with the overlying skin flap. The face-lift dissection is carried into the preplatysmal or subcutaneous neck dissection. The right hemi-forehead incision and subgaleal elevation is also performed.

4. Right lower eyelid subciliary incision/zygo-orbicular cheek dissection

- A subciliary incision is made with 6 to $7 \mathrm{~mm}$ of pretarsal orbicularis preserved with the initial skin flap, followed by a suborbicularis dissection performed by hugging the undersurface of the orbicularis muscle and continuing with a pre-periosteal dissection deep to the zygomaticus muscle, but maintaining the origin of the muscles. At this point, an arcus marginalis release is done, and excess fat may be resected if indicated. A canthopexy or canthoplasty is then performed depending on the preoperative assessment of canthal/lower lid position. A canthoplasty includes division of the complete lateral canthal tendon and reattachment to the periosteum or with osseous drill hole fixation to the lateral orbital rim. The septal reset over the orbital rim is accomplished using 5-0 Vicryl.

5. The left face, retroauricular, and hemi-forehead elevation is done in the same fashion as the right side. The left lower eyelid and cheek dissections and septal reset is done as in the right side.

6. An open coronal or hairline subgaleal forehead dissection with resection of the corrugator muscles is done. Endoscopic forehead lifts are not utilized.

7. The platysmal anterior borders are closed with a running "corset" closure using 3-0 Vicryl. If indicated, the fat is excised from the skin flap with scissors. Liposuction is rarely done.

\section{ClOsuRE}

- The right cheek lift closure is done following temporary fixation of the forehead with a towel clip to simulate a forehead lift. A laterally based orbicularis flap is passed under the lateral raphe and sutured to the periosteum of the lateral orbital rim with 4-0 Monocryl. The final skin trim is delayed until all closures are complete.

- The right face-lift closure is done using a subcuticular 3-0 Monocryl subcuticular retroauricular and a 4-0 Monocryl subcuticular retrotragal. The 


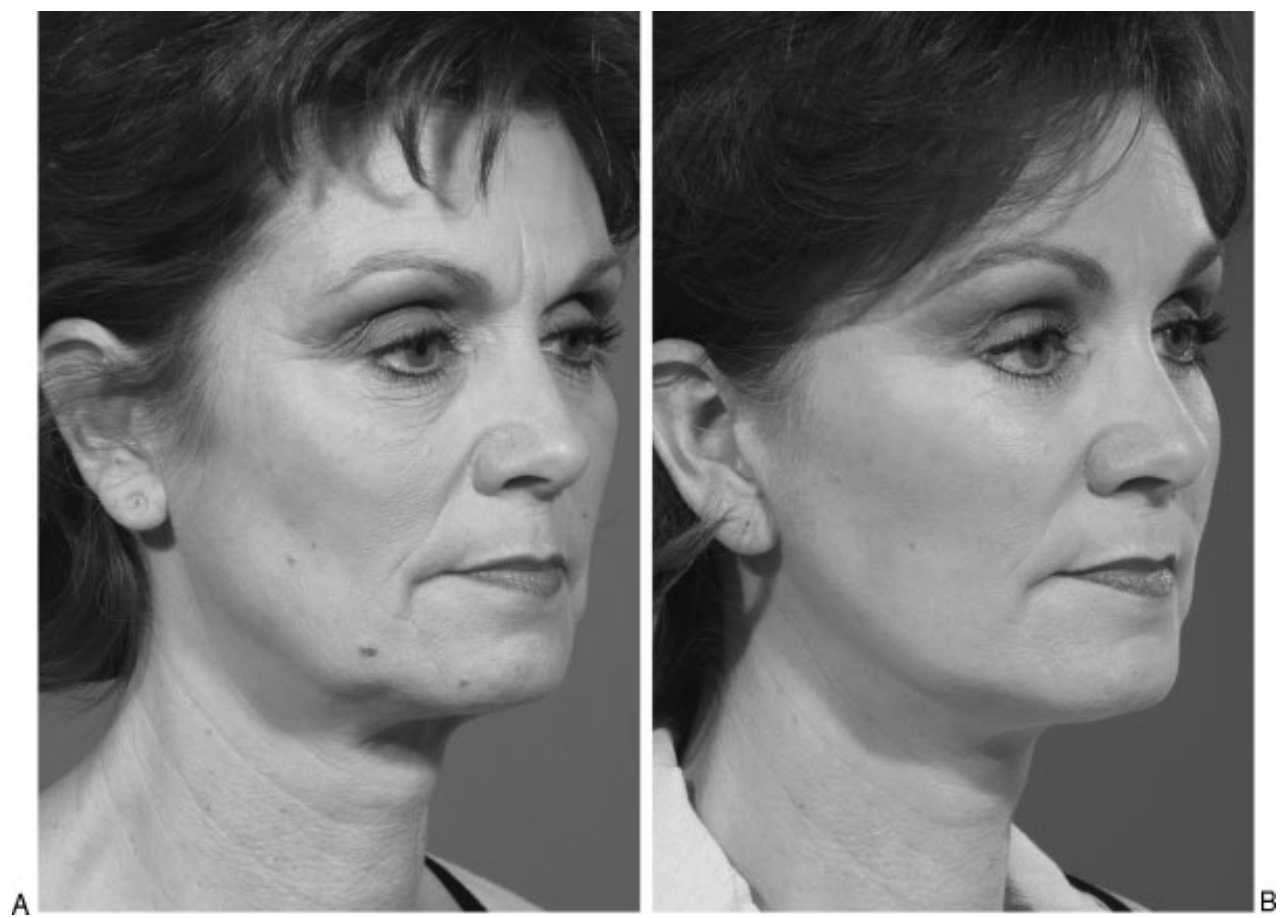

Figure 10 (A) Primary composite lift in a 60-year-old woman who has excellent skin and normal balance of facial structures. (B) Note the high cheek mass and postoperative facial harmony.

left cheek lift closure is done exactly as the right side was done, with a towel clip in the brow lift.

- The left face-lift closure is done in the same fashion as the right side.
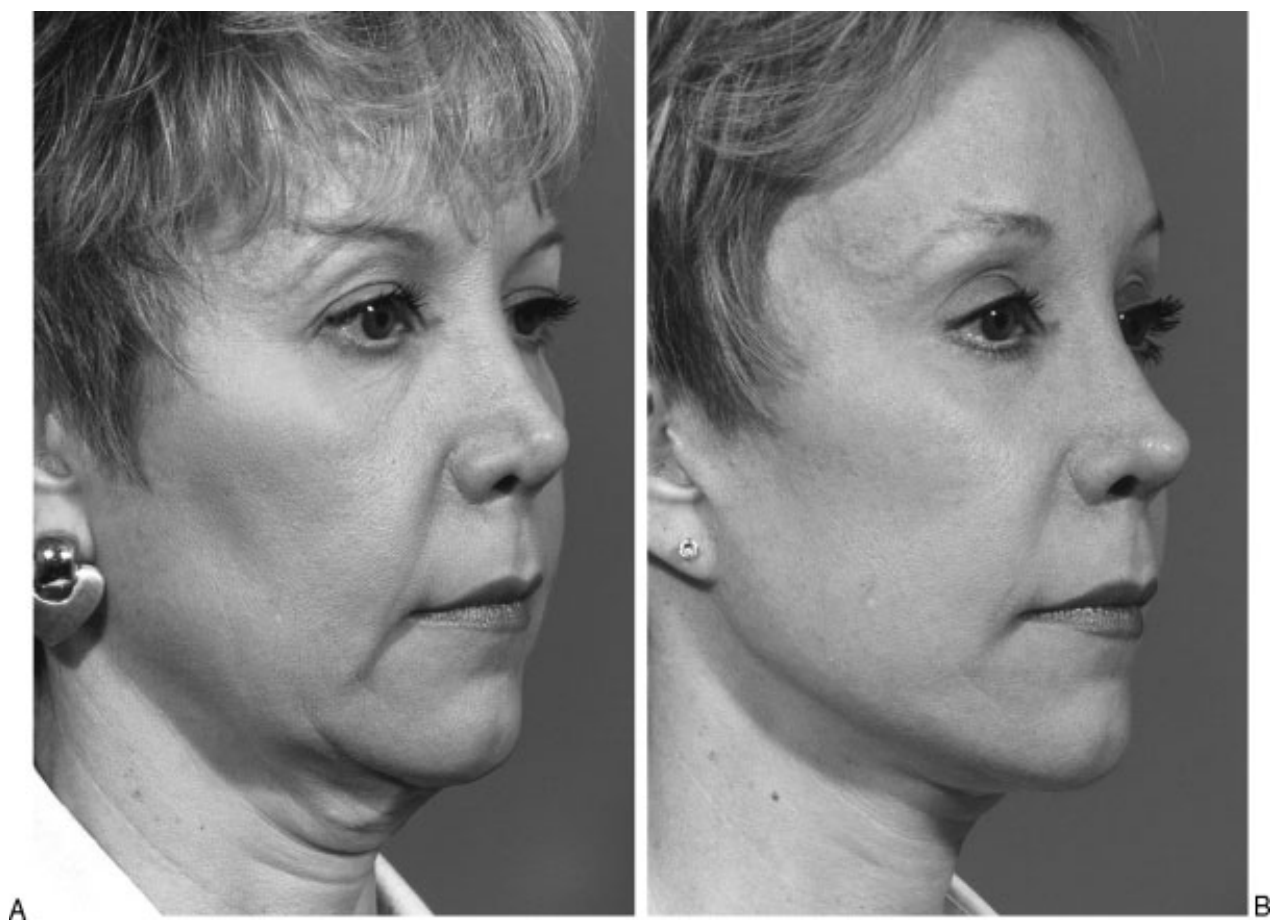

Figure 11 (A) Primary face-lift with previous rhinoplasty resulting in a foreshortened nose. The patient also has an overbite. (B) Composite face-lift with chin implant and secondary rhinoplasty for nasal lengthening. ${ }^{12}$
- The submental incision closure is done using 5-0 running nylon and a Jackson Pratt drain placement. The drain exits the right lateral lower neck using a trochar. 

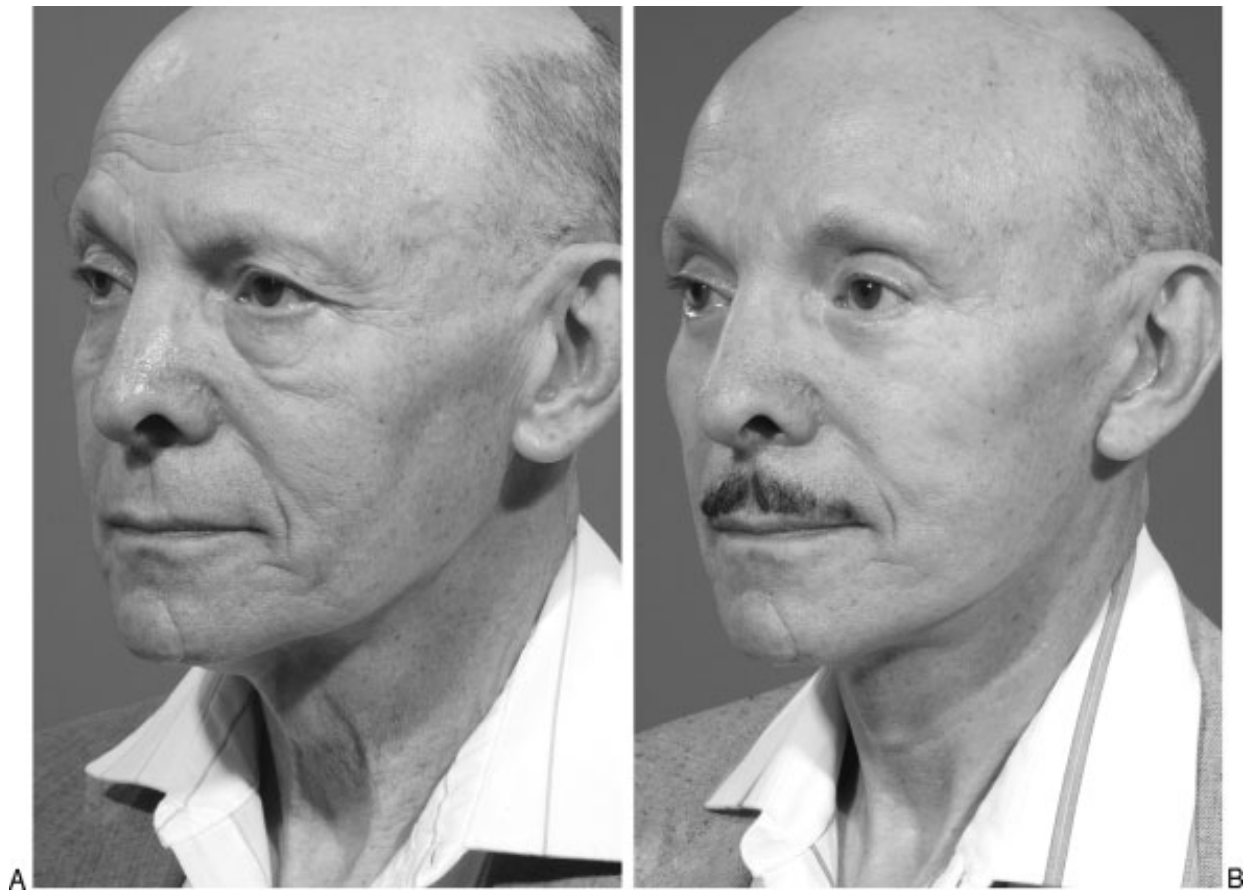

Figure 12 (A) Primary face-lift in a 73-year-old man with malar crescent deformity. (B) There has been a total correction of the excessive orbicularis and obliteration of the eyelid-cheek junction.

- Forehead lift closure is done using a running 3-0 Monocryl galeal closure and then staples in the skin.

- The lower eyelid skin trim is the last maneuver using 6-0 nylon for the dogleg and rapid-dissolving 6-0 catgut for the subciliary incision.

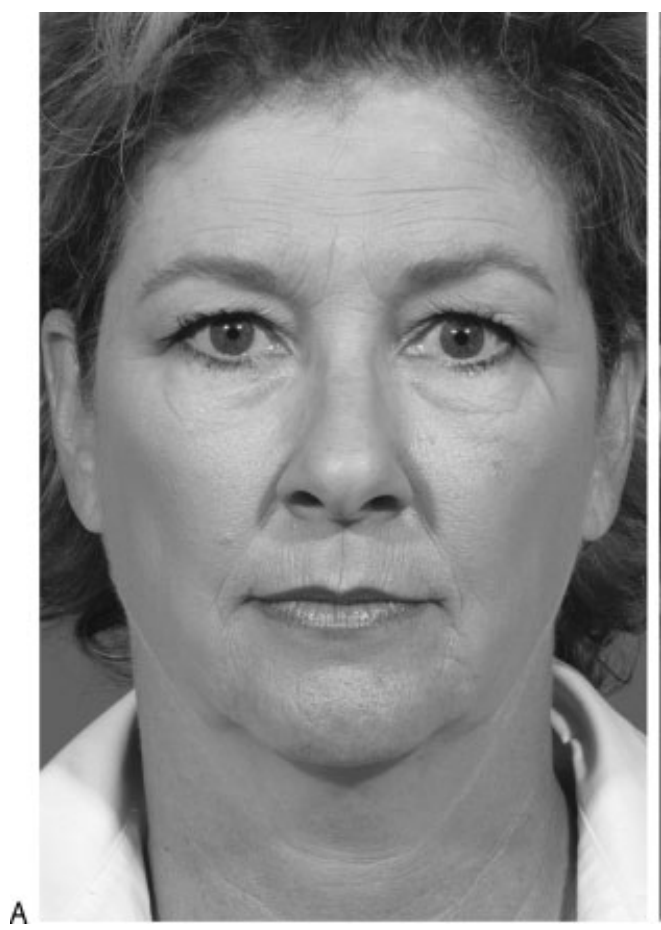

\section{OPERATIVE STEPS}

- The superior-medial vector cheek lift dictates an obligatory forehead lift done in a superomedial direction.

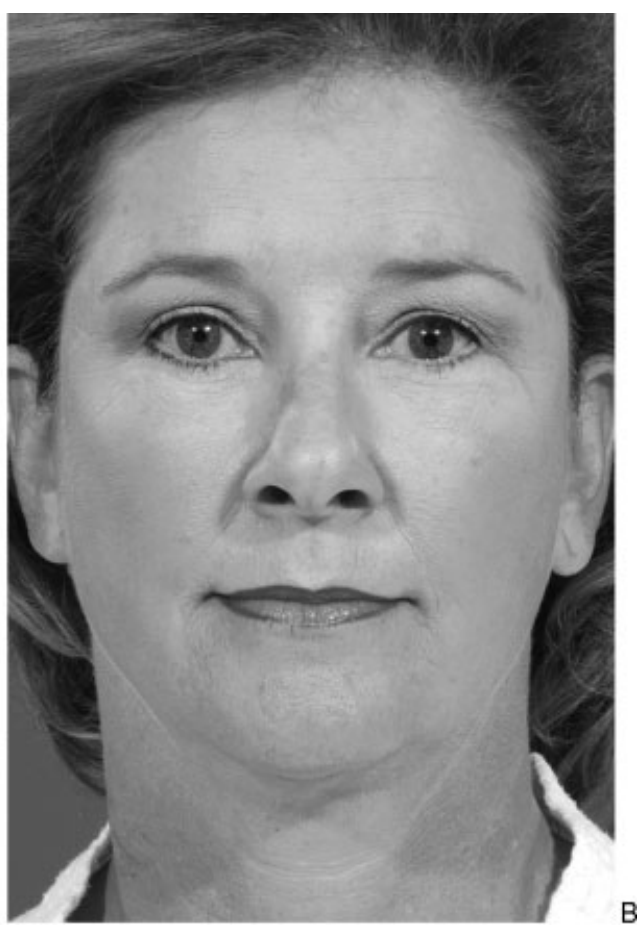

Figure 13 (A) Primary face-lift with excessive jowling from lower facial fat excess. (B) Open defatting of preplatysmal fat of the lower face creating a better jawline. Note the eyelid cheek junction. 


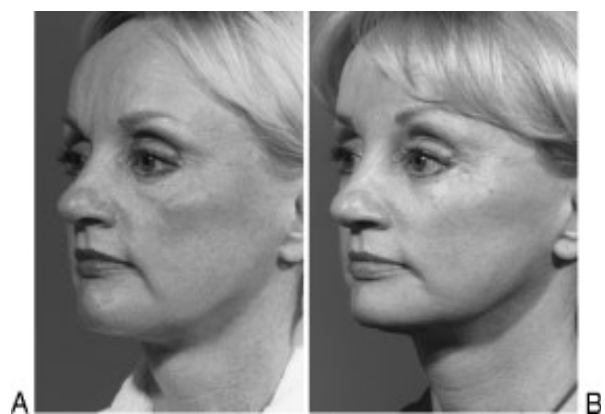

Figure 14 (A) Previous face-lift resulting in hollow eyes and a malar crescent. (B) Hairline forehead lift and composite face-lift for correction of hollow eyes and malar crescent deformity.

- A "deep plane" face-lift, which leaves all cheek fat with the skin, is done to elevate the malar fat with the skin so it can be moved with the skin toward the orbit.

- Depending on the anatomy, the orbital fat is preserved, and the septum is reset over the orbital rim after a canthoplasty is done. In some cases, excess fat must be excised.

- At closure, following intial fixation of the brow, the first tension is set on the zygo-orbicular flap attachment (i.e., lower eyelid/cheek lift). The second tension is set on the upper face attachment to the helix. The SMAS fixation if used is last, and must be countered and overcome by the zygo-orbicular closure to prevent an eventual lateral sweep. Frequently, the SMAS is not elevated.

- The tension on the obligatory forehead lift closure is superior-medial, and must be strong enough to prevent bunching at the lateral canthal area.

- A Jackson Pratt drain is left in the neck for 24 hours.

\section{RESULTS}

- Primary composite lift in a 60-year-old woman who has excellent skin and normal balance of facial structures. Note the high cheek mass and postoperative facial harmony (Fig. 10).

- Primary face-lift with previous rhinoplasty resulting in a foreshortened nose. The patient also has an overbite. Composite face-lift with chin implant and secondary rhinoplasty for nasal lengthening (Fig. 11). ${ }^{12}$

- Primary face-lift in a 73-year-old man with malar crescent deformity. There has been a total correction of the excessive orbicularis and obliteration of the eyelid-cheek junction (Fig. 12).

- Primary face-lift with excessive jowling from lower facial fat excess. Open defatting of preplatysmal fat of the lower face creating a better jawline. Note the eyelid cheek junction (Fig. 13).

- Previous face-lift resulting in hollow eyes and a malar crescent. Hairline forehead lift and composite
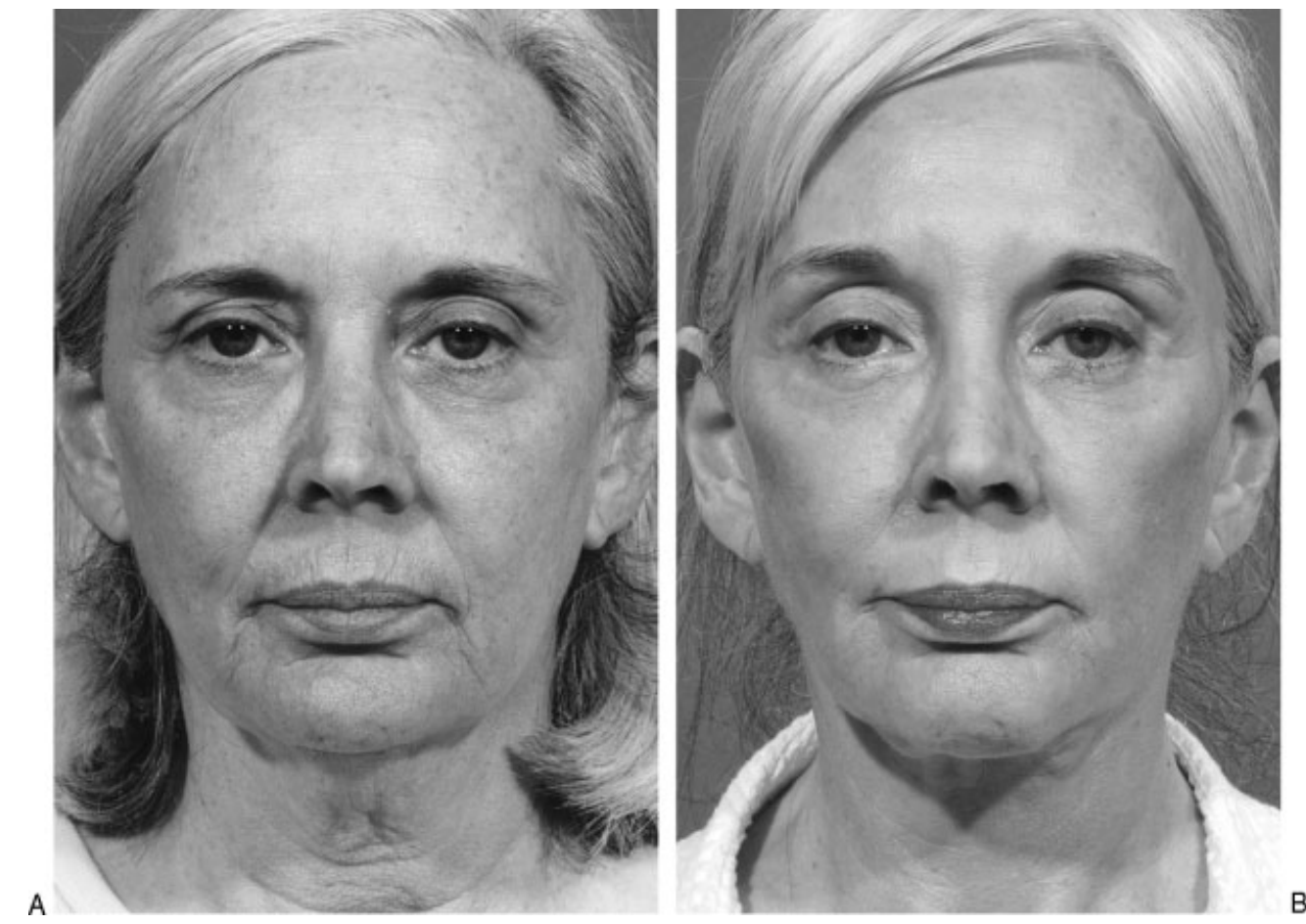

Figure 15 (A) Primary face-lift with panfacial aging including high forehead and hollow eyes. (B) Seven years following composite face-lift with hairline-lowering brow lift and dermabrasion of the upper lip. 

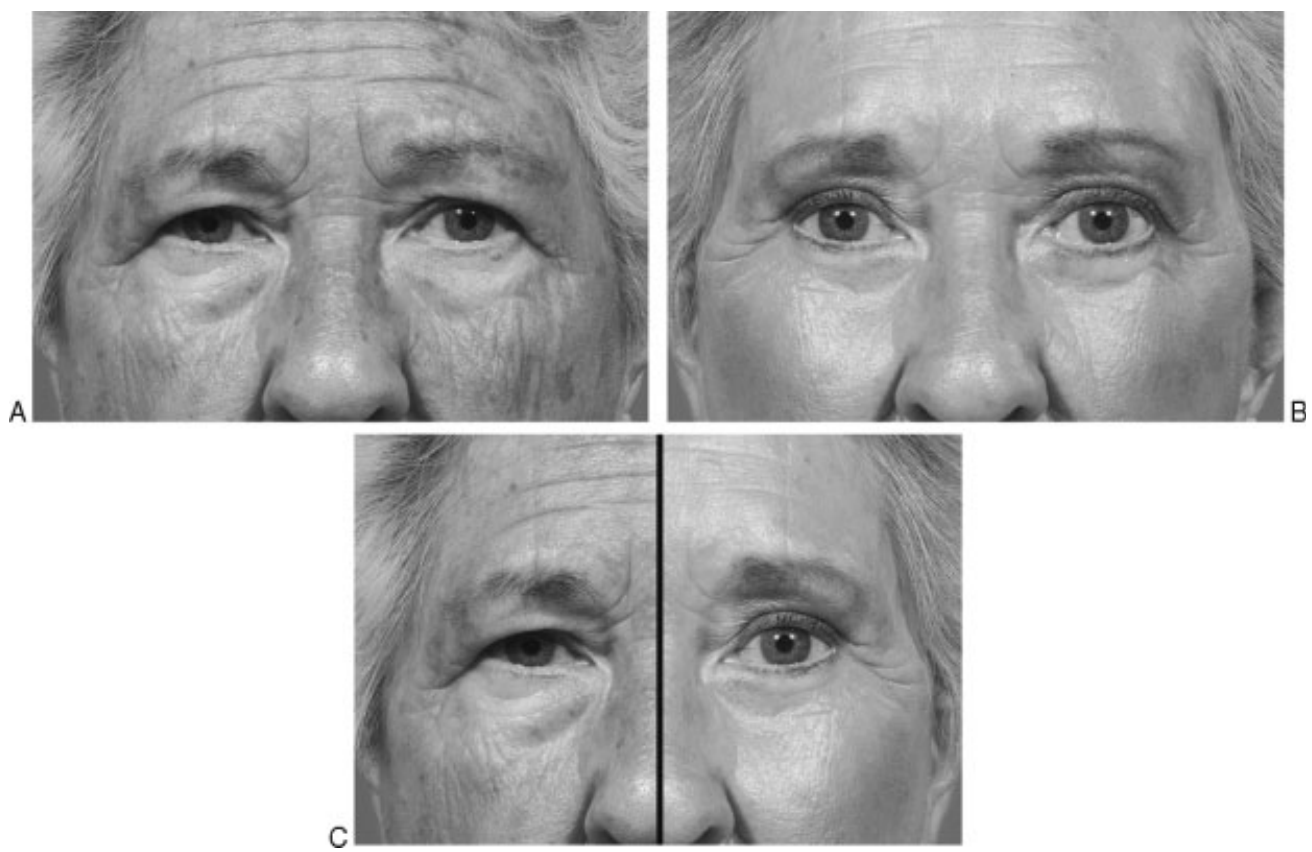

Figure 16 (A) Advanced aging of the periorbital area. (B) One year following periorbital rejuvenation with composite face-lift. (C) Side-by-side evaluation of eyelid results.

face-lift for correction of hollow eyes and malar crescent deformity (Fig. 14).

- Primary face-lift with panfacial aging including high forehead and hollow eyes. Seven years following composite face-lift with hairline-lowering brow lift and dermabrasion of the upper lip (Fig. 15).

- Advanced aging of the periorbital area. One year following periorbital rejuvenation with composite face-lift. Side-by-side evaluation of eyelid results (Fig. 16).

\section{Pitfalls and How to Correct}

The morbidity of this procedure compared with that of the conventional procedure is almost exclusively a question of symptoms concerning the eyelids. Because the movement of the orbicularis muscle dictates a surgical fixation to the orbital rim periosteum, there is a prolonged period of recovery compared with that of the conventional blepharoplasty where there is no tension on the skin-to-skin closure. ${ }^{13,14}$ The fibrosis created during the period of scar hypertrophy can cause difficulty with closure necessitating use of lubrication to prevent dryness. This may last until the patient passes the fourth postoperative week or beyond.

As with all more advanced procedures, there is a learning curve. This is particularly true when adjusting tension while suspending the tissues of the cheek. Lower eyelid retraction is always a concern, so that a close relationship with an ophthalmologist is helpful. The transcanthal canthoplasty helps prevent this problem. For minor cases of lower lid malposition, a simple tarsal strip is done under local anesthesia in the office. For more severe cases, a spacer graft may be necessary using Alloderm or Enduragen.

Because the face-lift flap is a musculocutaneous flap, the rich blood supply allows maximum tension to be exerted without fear of healing problems. Smokers are warned, but even when they do smoke, skin sloughs are rare because of the composite flap.

Because a complete facial rejuvenation is done, the patient is operated under general anesthesia, and an overnight stay is obligatory. During and after surgery, all precautions are taken including leg compression and clonidine patches for blood pressure control.

The goal of the composite face-lift is harmony of the entire face, with every area of the aging face compatibly rejuvenated with all other areas. Variations depend on each patient type. Patients with microgenia receive chin implants, and patients with high foreheads may have their forehead lift incisions at the hairline to lower the hairline. Variations of the phenol peel or dermabrasion are used for skin resurfacing when indicated.

\section{Postoperative Care}

The postoperative care is much the same as that for any face-lift operation, except that more care is needed for the eyes due to the fibrosis and healing of the periorbital area that result from the surgical attachment of the orbicularis to the periosteum of the lateral orbit. This causes tightness and thickening of the eyelids that prevent adequate closure for several weeks. On the day after surgery, the patient's hair is shampooed, and each day thereafter for several weeks. The patient leaves 
the hospital with no dressings and returns to the office on the fourth postoperative day for suture and staple removal. Lubrication of the eyes overnight with a nonmedicated ointment and drops during the day is used for several weeks to prevent cornea dryness. Pain is unusual after surgery, but medication is given as needed. Aerobic exercise is discouraged for 4 to 6 weeks, and if facial edema is obvious, then diuretics are prescribed.

\section{CONCLUSION}

The composite face-lift includes periorbital rejuvenation requiring a superior-medial face-lift vector that uses a zygo-orbicular flap for orbicularis repositioning coupled with a septal reset. It creates a harmonious rejuvenation in primary face-lift and rhytidectomy and can return harmony to faces distorted by previous face-lifts.

\section{REFERENCES}

1. Hamra ST. Composite rhytidectomy. Plast Reconstr Surg 1992;90:1-13

2. Hamra ST. Frequent face lift sequelae: hollow eyes and the lateral sweep: cause and repair. Plast Reconstr Surg 1998;102: 1658-1666

3. Choucair RJ, Hamra ST. Extended superficial musculaponeurotic system dissection and composite rhytidectomy. Clin Plast Surg 2008;35:607-622, vii
4. Hamra ST. A study of the long-term effect of malar fat repositioning in face lift surgery: short-term success but longterm failure. Plast Reconstr Surg 2002;110:940-951; discussion 952-959

5. Hamra ST. The tri-plane face lift dissection. Ann Plast Surg 1984;12:268-274

6. Hamra ST. The deep-plane rhytidectomy. Plast Reconstr Surg 1990;86:53-61; discussion 62-63

7. Hamra ST. Arcus marginalis release and orbital fat preservation in midface rejuvenation. Plast Reconstr Surg 1995;96:354-362

8. Hamra ST. The zygorbicular dissection in composite rhytidectomy: an ideal midface plane. Plast Reconstr Surg 1998;102:1646-1657

9. Lemmon ML, Hamra ST. Skoog rhytidectomy: a five-year experience with 577 patients. Plast Reconstr Surg 1980;65: 283-297

10. Hamra ST. Composite rhytidectomy. Plast Reconstr Surg 1992;90:1-13

11. Hamra ST. The role of the septal reset in creating a youthful eyelid-cheek complex in facial rejuvenation. Plast Reconstr Surg 2004;113:2124-2141; discussion 2142-2144

12. Hamra ST. Lengthening the foreshortened nose. Plast Reconstr Surg 2001;108:547-549

13. Hamra ST. Repositioning the orbicularis oculi muscle in the composite rhytidectomy. Plast Reconstr Surg 1992;90: 14-22

14. Hamra ST, Choucair RJ. Orbital aging and harmony in orbital rejuvenation. In: deCastro CC, Boehm K, Codner MA, eds. Techniques in Aesthetic Plastic Surgery Series: Midface Surgery. Oxford, UK: Elsevier; 2008 